\title{
Pylephlebitis presenting as spontaneous coronary sinus thrombosis: a case report
}

Michael A. Hart ${ }^{1 *}$ and Mengistu A. Simegn ${ }^{2}$

\begin{abstract}
Background: Coronary sinus thrombosis is a rare phenomenon. When identified, it most often is a complication of infective endocarditis or procedural intervention. We present an unusual and unreported case of spontaneous coronary sinus thrombosis as embolic sequela of an intra-abdominal infectious process.

Case presentation: We report a case of a 61-year-old white woman with a history of end-stage renal disease on hemodialysis, paroxysmal atrial fibrillation not on long-term systemic anticoagulation, and history of recurrent diverticulitis that presented with acute onset abdominal pain and nausea. Computed tomography of her abdomen and pelvis with intravenous contrast was negative for acute intra-abdominal pathology, but incidentally identified an oval-shaped filling defect at the ostium of the coronary sinus suspicious for thrombus or mass which was confirmed on subsequent transesophageal echocardiogram. In light of her concomitant transaminitis but otherwise negative workup, the mass was believed to be thromboembolic in nature, originating within the hepatic venous system as a manifestation of recurrent diverticulitis with associated pylephlebitis and ultimately lodging into the coronary sinus. With the newly detected thrombus and history of paroxysmal atrial fibrillation, she was started on warfarin for therapeutic systemic anticoagulation that resolved her clot by 3-month follow up.

Conclusions: Although coronary sinus thrombosis is rare, a high index of suspicion and close scrutiny of the venous system in patients with intra-abdominal infectious processes would prevent delay in management of this potentially serious complication. The discussion of this case highlights the anatomy of the cardiac venous system, the pathophysiology of thrombus formation, and the utility of transesophageal echocardiography in confirming a diagnosis and assessing treatment efficacy.
\end{abstract}

Keywords: Coronary sinus, Thrombus, Transesophageal echocardiography

\section{Background}

Coronary sinus thrombosis (CST) is an uncommon complication of instrumentation or procedures in the heart or great vessels. Here we present a case of spontaneous CST believed to be a manifestation of pylephlebitis with transient hepatic vasculature thrombus traveling to and lodging in the coronary sinus (CS). To the best of our knowledge, there are no reported cases of spontaneous CST as sequela of intra-abdominal infectious processes. The discussion highlights the anatomy of the cardiac venous system, the pathophysiology of thrombus formation, and treatment options for this rare but potentially serious and fatal condition.

\footnotetext{
* Correspondence: Michael.Hart@hcmed.org

${ }^{1}$ General Internal Medicine, Hennepin County Medical Center, 701 Park

Avenue, Minneapolis, MN 55415, USA

Full list of author information is available at the end of the article
}

\section{Case presentation}

A 61-year-old white woman was hospitalized for acute onset abdominal pain and nausea. She had been well until 1 day earlier when she began to have continuous nausea, non-projectile recurrent emesis of ingested matter, anorexia, and epigastric abdominal pain.

Her past medical history included end-stage renal disease (ESRD) and two previous renal transplants secondary to immunoglobulin A (IgA) nephropathy on chronic immunosuppressive therapy with subsequent graft failure requiring return to hemodialysis for the past 3 years, non-ischemic dilated cardiomyopathy with nadir ejection fraction (EF) of 15 to 20\%, hypertension, paroxysmal atrial fibrillation not on chronic systemic anticoagulation, mild to moderate non-obstructive coronary artery disease, and history of recurrent diverticulitis. She is unemployed, a former tobacco smoker quitting in 1993, she denied 
alcohol or recreational drug use, has no significant family history, and is allergic to angiotensin-converting enzyme (ACE) inhibitors (cough). Home medications include aspirin, atorvastatin, carvedilol, losartan, clonidine, and hydralazine.

On physical examination, she appeared acutely sick, afebrile, and normotensive with normal pulse oxygen saturation. A cardiovascular examination was significant for regular rate and rhythm with a 2/6 systolic murmur heard throughout the precordium. Pulmonary and neurologic examinations were unremarkable; extremity examination was notable for left upper extremity fistula with palpable thrill and mild pitting edema in bilateral lower extremities extending to mid-shin. Her abdomen was soft, mildly distended, and tender to palpation in the epigastrium without Murphy's sign or rebound tenderness.

Hematology and chemistry panels were significant for neutrophilic leukocytosis $\left(14.5 \mathrm{k} / \mathrm{mm}^{3}\right)$, mild normocytic anemia $(9.8 \mathrm{~g} / \mathrm{dL})$, hyponatremia $(128 \mathrm{mEq} / \mathrm{L})$, and hyperkalemia $(6.1 \mathrm{mEq} / \mathrm{L})$. Hepatic panel showed: transaminitis with aspartate aminotransferase (AST) 1078 and alanine aminotransferase (ALT) $660 \mathrm{IU} / \mathrm{L}$; with normal total and fractional bilirubin; and mildly elevated alkaline phosphatase (113 IU/L). Evaluations for acetaminophen toxicity, infectious and autoimmune hepatitis, primary biliary cirrhosis, primary sclerosing cholangitis, and Wilson's disease were unremarkable. Telemetry and electrocardiogram illustrated known paroxysmal atrial fibrillation.

Computed tomography (CT) of her abdomen and pelvis with intravenous contrast was negative for acute intra-abdominal pathology including thrombosis of the hepatic veins, but incidentally identified an oval-shaped filling defect at the junction of the right atrium and CS suspicious for thrombus or mass (Fig. 1a). Transesophageal echocardiogram (TEE), a better imaging modality for tissue characterization and assessment of the right atrium and CS, demonstrated a $1.5 \times 1.5 \mathrm{~cm}$ echogenic globular mass at the ostium of the CS consistent with thrombus (Fig. 1b). The left ventricle was normal in size, but systolic function was mildly depressed.

With the newly detected thrombus and history of paroxysmal atrial fibrillation, our patient was started on warfarin for therapeutic systemic anticoagulation. At her 3-month out-patient visit, her liver enzymes had normalized, and TEE and gated cardiac CT, optimal imaging modality for assessment of the entire cardiac venous system, showed complete resolution of the CS thrombus (Fig. 2a and b). In light of paroxysmal atrial fibrillation and development of spontaneous CST, the decision was made at 6-month follow up to continue anticoagulation long term. Her subsequent clinical course was remarkable for development of polymicrobial intra-abdominal abscess (Enterococcus faecalis, Escherichia coli, and Candida albicans) 8 months after the index event requiring drain placement and 4 weeks of antibiotic therapy. She was ultimately discharged to sub-acute rehabilitation with plan for a partial colectomy to be done in the coming months after improvement in her functional capacity.

\section{Discussion}

Here we present an unusual and unreported case of spontaneous CST as embolic sequela of an intra-abdominal infectious process. Thrombosis of the CS is an exceedingly rare event, with the majority of cases reported in the literature occurring in the setting of invasive procedures or infection. Development of CST in the absence of instrumentation is even less common, and believed to be secondary to underlying prothrombotic states [1-3]. Despite this, the potential complications of CST can be
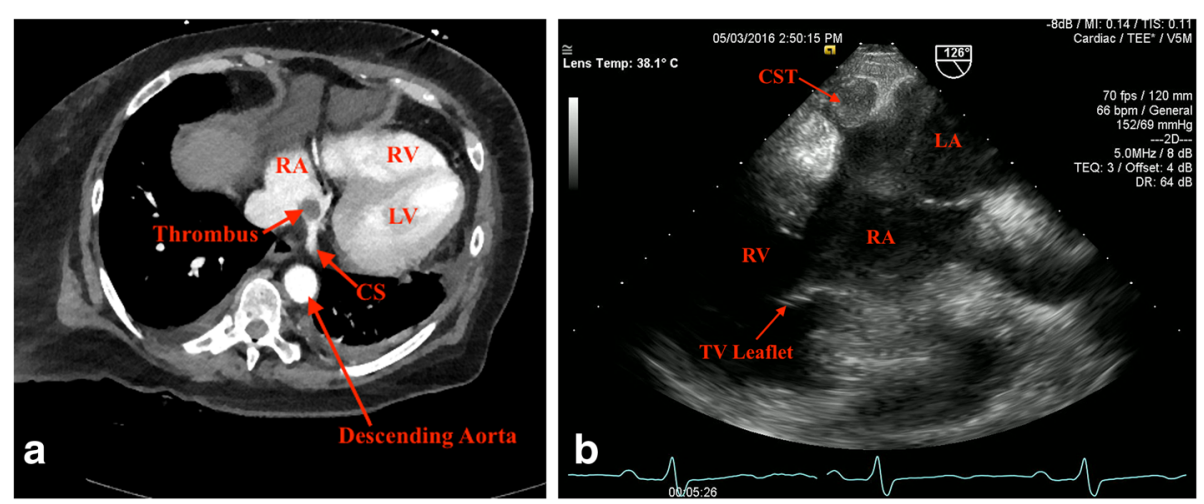

Fig. 1 a Chest computed tomography with contrast at the level of coronary sinus showing an oval-shaped filling defect at the junction of the right atrium and coronary sinus suggestive of coronary sinus thrombus. b Transesophageal echocardiogram at the gastroesophageal junction positioned at $126^{\circ}$ demonstrating a $1.5 \times 1.5 \mathrm{~cm}$ echogenic globular mass at the ostium of the coronary sinus. CS coronary sinus, CST coronary sinus thrombus, $L A$ left atrium, $L V$ left ventricle, $R A$ right atrium, $R V$ right ventricle, $T V$ tricuspid valve 


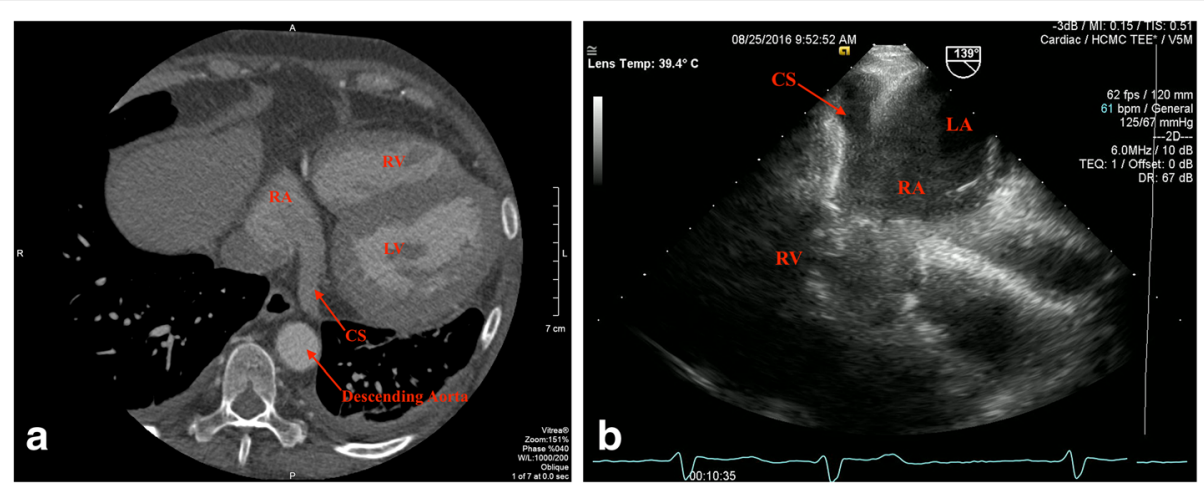

Fig. 2 a Gated cardiac computed tomography at the coronary sinus level showing patent coronary sinus with resolution of the prior filling defect after 3 months of systemic anticoagulation. $\mathbf{b}$ Transesophageal echocardiogram at the gastroesophageal junction positioned at $139^{\circ}$ confirmed patent coronary sinus. CS coronary sinus, LA left atrium, $L V$ left ventricle, $R A$ right atrium, $R V$ right ventricle

catastrophic and include myocardial infarction, cardiac tamponade, sudden cardiac arrest, and death [1, 4-6].

The CS is the largest venous collection system of the heart, accounting for over half of the total cardiac venous return. Its anatomical position at the posterior aspect of the coronary sulcus places it in close proximity to the inferior vena cava (IVC) through which it communicates directly with the rest of the body's venous system. Our patient's history of paroxysmal atrial fibrillation, ESRD, and chronic immunosuppressive therapy with recurrent diverticulitis is likely to have contributed to her overall inflammatory and procoagulant state. In this context, we suspect she developed congestive hepatitis from pylephlebitis and transient thrombosis of the hepatic vein, which then migrated to the right atrium and lodged in the CS ostium. This mechanism explains her presentation of acute abdominal pain, nausea, vomiting, transaminitis, and right atrial mass.

Pylephlebitis is a rare and serious complication of intraabdominal infection and is most commonly associated with diverticulitis $[7,8]$. Thrombotic communication between the hepatic and cardiac venous systems has been demonstrated in the literature on one other occasion, but again was in the setting of instrumentation [5]. Both mechanical and pharmacologic treatment of this condition have been successful in the past, with the latter strategy proving effective in resolving our patient's CS thrombus and hepatitis $[4,9]$.

\section{Conclusions}

Thrombus formation within the CS is extremely rare, with the majority of cases occurring as a result of instrumentation or prothrombotic states. Clinicians should be cognizant of the communication between the CS and the rest of the body's venous system and the potential for serious complication when systemic thrombosis leads to embolization to the CS. Once identified, initiation of mechanical or pharmacologic treatment should follow promptly to avoid the potentially deadly manifestations of this phenomenon.

\section{Abbreviations \\ ACE: Angiotensin-converting enzyme; ALT: Alanine aminotransferase; AST: Aspartate aminotransferase; CS: Coronary sinus; CST: Coronary sinus thrombosis; CT: Computed tomography; EF: Ejection fraction; ESRD: End- stage renal disease; IgA: Immunoglobulin A; IVC: Inferior vena cava; TEE: Transesophageal echocardiogram}

\section{Acknowledgements \\ Not applicable}

Funding

There is no funding to report for this article.

Availability of data and materials

Data sharing not applicable to this article as no datasets were generated or analyzed during the current study.

Authors' contributions

$\mathrm{MH}$ performed the literature search, and composed the case report. MS served as advisor and editor for this case report. Both authors read and approved the final manuscript.

Ethics approval and consent to participate

Not applicable

\section{Consent for publication}

Written informed consent was obtained from the patient for publication of this case report and any accompanying images. A copy of the written consent is available for review by the Editor-in-Chief of this journal.

Competing interests

The authors declare that they have no competing interests.

\section{Publisher's Note}

Springer Nature remains neutral with regard to jurisdictional claims in published maps and institutional affiliations.

\section{Author details}

${ }^{1}$ General Internal Medicine, Hennepin County Medical Center, 701 Park Avenue, Minneapolis, MN 55415, USA. ${ }^{2}$ Cardiology, Hennepin County Medical Center, 701 Park Avenue, Orange 5, Minneapolis, MN 55415, USA. 
Received: 8 June 2017 Accepted: 28 September 2017

Published online: 02 November 2017

\section{References}

1. Bapat VN, Hardikar AA, Porwal MM, Agrawal N, Tendolkar AG. Coronary sinus thrombosis after cannulation during cardiopulmonary bypass. Ann Thorac Surg. 1996;62:1506-7.

2. Floria M, Negru D, Antohe I. Coronary sinus thrombus without spontaneous contrast. J Thromb Thrombolysis. 2016;42:421-2. doi:10.1007/s11239-0161369-9.

3. Frogel JK, Weiss SJ, Kohl BA. Transesophageal echocardiography diagnosis of coronary sinus thrombosis. Anesth Analg. 2009;108(2):441-2. doi:10.1213/ ane.0b013e31818f61e3.

4. Güvenç TS, Karakuş G, Karabaǧ Y, Özen K, Balci B. Thrombus at ostium of coronary sinus discovered during evaluation for cardiac resynchronization therapy. Echocardiography. 2012;29(7):E176-7. doi:10.1111/j.1540-8175.2012. 01693.x.

5. Figuerola M, Tomas MT, Armengol J, Bejar A, Adrados M, Bonet A. Pericardial tamponade and coronary sinus thrombosis associated with central venus catheterization. Chest. 1992;101(4):1154-5.

6. Ramsaran EK, Sadigh M, Miller D. Sudden cardiac death due to primary coronary sinus thrombosis. South Med J. 1996:89(5):531-3.

7. Choudhry AJ, Baghdadi YMK, Amr MA, et al. Pylephlebitis: a review of 95 cases. J Gastrointest Surg. 2016;20(3):656-61. doi:10.1007/s11605-015-2875-3. Pylephlebitis.

8. Wong K, Student M, Weisman DS, Patrice K. Pylephlebitis: a rare complication of an intra-abdominal infection. J Community Hosp Intern Med Perspect. 2013;3(2):1-4.

9. Huang $H$, Yeh $\mathrm{K}$. Successful treatment of acute coronary sinus thrombosis utilizing a Judkins right catheter in a patient receiving cardiac resynchronization therapy. Europace. 2009;11(12):1674. doi:10.1093/ europace/eup319.

\section{Submit your next manuscript to BioMed Central and we will help you at every step:}

- We accept pre-submission inquiries

- Our selector tool helps you to find the most relevant journal

- We provide round the clock customer support

- Convenient online submission

- Thorough peer review

- Inclusion in PubMed and all major indexing services

- Maximum visibility for your research

Submit your manuscript at www.biomedcentral.com/submit 